\title{
Tratamiento informativo y competencias mediáticas sobre la COVID-19 en Ecuador
}

\author{
Informative treatment and media competencies on COVID-19 in \\ Ecuador
}

\author{
Mullo López, A., de-Casas-Moreno, P. y Balseca Mera, J. M. ${ }^{1}$ \\ Recibido: 12-09-2020 - Aceptado: 27-01-2021 \\ https://doi.org/10.26441/RC20.1-2021-A8
}

\begin{abstract}
RESUMEN: La sobresaturación informativa, desinformación y noticias falsas ha propiciado la necesidad de reforzar la alfabetización mediática. Con el decreto de emergencia sanitaria mundial producido por la aparición del CoViD-19, los usuarios de medios tradicionales y digitales se encuentran en una búsqueda informativa constante sobre el tema. El presente estudio analiza las prácticas comunicativas de los usuarios de los medios de comunicación y su percepción en torno a la información que reciben sobre el coronavirus. Para ello, se ha realizado una investigación cuantitativa con ayuda de un cuestionario autoadministrado a un total de 2.090 ecuatorianos. Además, se hace uso de la técnica de ingeniería inversa de datos para analizar las temáticas y plataformas tradicionales y digitales a las que frecuentemente accede la ciudadanía para informarse. Los resultados indican que existe una preferencia por la información publicada en plataformas de la Web 2.0: referencias a directrices sanitarias y una posible vacuna para enfrentar la enfermedad. También, se identifica una pérdida de confianza en los medios de comunicación tradicionales y en las fuentes gubernamentales, en tanto que la ciudadanía le da mayor credibilidad a las noticias compartidas por amigos y familiares. Por tanto, se concluye que es necesario desarrollar estrategias focalizadas a la diversidad de grupos sociales. Esto se puede llevar a cabo mediante un trabajo articulado entre los organismos de salud, científicos de las universidades ecuatorianas, medios de comunicación (tradicionales - digitales) y la participación ciudadana (prosumer) por medio de propuestas comunicacionales interactivas. Estos permitirán contrarrestar la infodemia mediante la alfabetización mediática y la responsabilidad digital para posicionar la información basada en evidencia científica.
\end{abstract}

Palabras clave: CoViD-19; coronavirus; pandemia; infodemia; medios de comunicación; Web 2.0; política de la comunicación; opinión pública; competencia mediática; estrategias de comunicación.

ABSTRACT: Information overload, misinformation and false news have led to the need to strengthen media literacy. With the global health emergency decree produced by the appearance of CoViD-19, users of traditional and digital media are in a constant search for information on the subject. This study analyzes the communication practices of media users and their perception of the information they receive about the coronavirus. To this end, quantitative research was done with the help of a self-administered questionnaire to a total of 2,090 Ecuadorians. In addition, use is made of the technique of reverse engineering of data

\footnotetext{
${ }^{1}$ Alex Mullo López es Magister en Comunicación periodística, institucional y empresarial por la Universidad de los Andes: Ambato (Ecuador), Docente y Director de la carrera de Comunicación de la Universidad Técnica de Cotopaxi (Ecuador). Su línea de investigación está centrada en la educomunicación y la convergencia digital. alex. mullo@utc.edu.ec, https://orcid.org/0000-0001-9876-9210
}

Patricia de Casas Moreno es Doctora en Comunicación en la rama de Alfabetización Mediática por la Universidad de Huelva. Profesora Ayudante Doctora de la Universidad Antonio de Nebrija y la Universidad Internacional de la Rioja. Su línea de investigación está centrada en la calidad de los medios de comunicación, las nuevas narrativas y estudios eduocomunicativos. pcasas@ @ebrija.es, https://orcid.org/0000-0003-1205-8106

Johana Maribel Balseca Mera es Máster en Comunicación y Docente en la Facultad de Jurisprudencia y Ciencias Sociales de la Universidad Técnica de Ambato (Ecuador). Su línea de investigación está centrada en la comunicación corporativa y los estudios educomunicativos. jm.balseca@uta.edu.ec, https://orcid.org/0000-0002-3535-9262 
to analyze the topics and traditional and digital platforms that citizens frequently access to get information. The results indicate that there is a preference for the information published on Web 2.0 platforms: topics related to health guidelines and a possible vaccine to face the disease. Also, a loss of confidence in traditional media and government sources is identified, while citizens give greater credibility to news shared by friends and family. Therefore, it is concluded that it is necessary to develop strategies focused on the diversity of social groups. This can be done by working among health organisms, scientists from Ecuadorian universities, media (traditional - digital) and citizen participation (prosumer) through interactive communicational proposals. These will make it possible to counteract the infodemic through media literacy and digital responsibility to position information based on scientific evidence.

Keywords: CoViD-19; coronavirus; pandemic; infodemic; media; Web 2.0; communication policy; public opinión; media literacy; communication strategies.

\section{Introducción}

La nueva enfermedad, conocida como coronavirus o CoViD-19, fue notificada por primera vez el 31 de diciembre del 2019 en la ciudad de Wuhan (China). Posteriormente, el 30 de enero del 2020, la Organización Mundial de la Salud (OMS) declara alerta mundial sanitaria debido a la epidemia. A partir de ahí, se han presentado diversos casos en todo el mundo. En el caso de América Latina surge la primera persona contagiada en Brasil el 26 de febrero del 2020. Particularmente en Ecuador, la llegada del virus se registra por medio de un caso importado de España, reportado por el Ministerio de Salud el 28 de febrero del 2020. En este punto, con más de 114 millones de contagios en 114 países y 2,54 millones de personas fallecidas, la OMS proclama al CoViD-19 como pandemia el 11 de marzo del 2020 (BBC News, 2020).

La epidemia se muestra en la agenda mediática mundial a partir del primer caso en Wuhan. El coronavirus ya era conocido desde 1960, siendo una familia de virus causantes del $15 \%$ de los resfriados comunes (Ahmed et al., 2020), así como de enfermedades más graves como el síndrome respiratorio de Oriente Medio (MERS- CoV), el síndrome respiratorio agudo severo (SRAS) y el nuevo coronavirus denominado CoViD-19. A raíz de la aparición del nuevo genoma secuenciado, la preocupación de la población mundial se ha visto reflejada en las plataformas digitales. Por ejemplo, en España el tema fue tendencia en Twitter tras el anuncio del primer caso registrado en su territorio el 1 de febrero del 2020 (Chen, Lerman \& Ferrara, 2020). Situación similar ocurrió en Ecuador. En cuanto a las afectaciones económicas, estas tuvieron incidencia en los escenarios mediáticos de Europa y Estados Unidos a finales de marzo del 2020, evidenciado por el impacto del confinamiento (Odriozola-Chéné et al., 2020).

A la intranquilidad de cómo tratar el virus se ha sumado la imperiosa necesidad de contrarrestar la información falsa, referente a rumores, bulos, mitos, como por ejemplo tratamientos o medicamentos que curan la enfermedad o teorías conspirativas, que hacen referencia a que el CoViD-19 fue creado en un laboratorio en China con el fin de reducir la población mundial (Andreu-Sánchez \& Martín-Pascual, 2020; Pulido et al., 2020). De esta forma, la infoxicación y sobresaturación informativa dificulta que la sociedad conozca las noticias correctas para enfrentar la enfermedad, provocando ansiedad en la población (Naeem \& Bhatti, 2020). De ahí, que la abundancia de información es el primer problema que se origina debido a la accesibilidad a Internet (Aguaded \& Rodríguez-Romero, 2015) y con ello, la dubitación entre la calidad informativa y las fakes news. Por ende, no hablamos solo del producto final como -la información- sino de las circunstancias internas y externas del medio que las produce y publica (Romero-Rodríguez, de-Casas-Moreno \& Torres-Toukoumidis, 2016). Tanto es así, que múltiples países han instaurado el fact-checking para verificar noticias falsas y manipulación en los posts existentes en la Red, intentando así contribuir con el control de la desinformación actual e instando al receptor a ser más crítico y activo en la confirmación de información antes de masificarla con sus redes en las plataformas (Lotero-Echeverri, Romero-Rodríguez \& Pérez-Rodríguez, 2018). De esta manera, se evita que los comportamientos 
de los analfanautas y las competencias críticas se vean influenciadas por las competencias mediáticas en el ecosistema digital con base en una temática viralizada (Romero-Rodríguez, de-Casas-Moreno \& Caldeiro-Pedreira, 2018).

Por otro lado, a esta problemática la OMS la define como infodemia, impidiendo que las personas tomen las medidas adecuadas para enfrentar eficazmente la enfermedad y provocando conductas peligrosas, como por ejemplo, la automedicación de sustancias nocivas generadas por el temor y la agitación (OMS, 2020). Además, esto reduce la eficacia de los programas y campañas de los organismos de salud pública, que buscan la concienciación y el bienestar de los ciudadanos (Pulido et al., 2020). En el caso de el Congo, la creencia en informes erróneos, durante el brote del virus del ébola del año 2018, provocó menos conductas preventivas por parte de la población (Brainard \& Hunter, 2020). Por tanto, la infodemia no es un término nuevo, debido a que los brotes de desinformación son tan antiguos como Internet, ya que desde 1997 se publicaban investigaciones referentes a la falsa información médica en la Red (Eysenbach, 2020).

Bajo este escenario de emergencia sanitaria, la difusión de noticias falsas y contenido no verificable sobre el CoViD-19, se está propagando a un ritmo preocupante en los medios de comunicación (Kouzy et al., 2020). Esto represenra una grave amenaza para el orden público y la empatía social (Rinaldi, 2020). De ahí la importancia y responsabilidad que tienen los medios de comunicación para mantener debidamente informada a la población sobre la prevención de enfermedades (Mejia et al., 2020). No obstante, la ciudadanía ahora ya no se informa a través de un solo medio de comunicación, sino más bien, su interés va dirigido hacia el contenido, combinando diferentes medios tradicionales y digitales para obtener la información necesaria (Casero-Ripolles, 2020). Es así como en tiempos de emergencia sanitaria, se evidencia la preferencia por los medios digitales, concretamente, las redes sociales, debido a la actualización permanente y constante del contenido (Masip et al., 2020).

El usuario de este tipo de plataformas tiene el compromiso de seleccionar las fuentes informativas que considere importante y confiable (Eysenbach, 2020). Sobre este tema, algunos expertos como Ahmad, Murad y Gardner (2020) indican que en la Web 2.0, muchas veces, las personas no pueden discernir si la información es verdadera o falsa, prevaleciendo en primer lugar la demonización sobre la situación de la pandemia. El grado de credibilidad de las noticias de los medios sociales no radica en la fuente informativa, sino más bien, en la confianza que se tenga en la persona que comparte dicha noticia (Sterrett et al., 2019). Es ahí donde cobra relevancia el usuario, que no solo consume información, sino que también es capaz de producir y generar contenido informativo. A este tipo de usuario se le denomina prosumer, término propuesto por Alvin Toffler (1981) en su libro la tercera ola. A partir de esta premisa, la veracidad de las publicaciones en redes sociales no se basa únicamente en la realidad que se transmite en el contenido, sino en la autoría (prosumer) de quien las construye (Catalina-García, Sousa \& Silva-Sousa, 2019). En este orden de ideas, figura en un segundo escenario la espectacularización en la reconstrucción mediática y reiterada de hechos noticiosos, generando un agotamiento cognitivo en el perceptor y asimilando el tema como parte de la -normalidad- (Torres-Toukoumidis et al., 2017). De este modo, aumenta la necesidad de que los gobiernos locales generen infodemiología y vigilen el comportamiento de búsqueda de información de salud en Internet (Effenberger et al., 2020).

Por otra parte, Bonnet y Rosenbaum (2020) afirman que el favoritismo por las redes sociales y los medios nativos digitales, frente a los medios de comunicación tradicionales como la radio, prensa o la televisión, pueden aumentar el clima de incertidumbre sobre la veracidad de las noticias. Pero ¿por qué la gente tiene preferencia en las fuentes informativas de la Web 2.0?. A criterio de Casero-Ripolles (2020), existe una pérdida de credibilidad en los medios tradicionales, originando más desinformación. Sin embargo, las plataformas Web 2.0 no serían las únicas responsables de la infodemia, ya que las autoridades gubernamentales también están contribuyendo a la desinformación 
debido a la emisión de noticias sanitarias no verificadas (Tangcharoensathien et al., 2020). Hay que destacar, por ejemplo, las declaraciones del presidente de los Estados Unidos, Donald Trump, quien aseguraba que la hidroxicloroquina era un medicamento probado para tratar el coronavirus. Estas expresiones fueron replicadas por los medios tradicionales. Más tarde los investigadores confirmaron que no funciona contra el virus y que incluso puede causar problemas cardiacos. Otro caso, de desinformación relevante publicado en redes sociales por el presidente anteriormente citado, afirmaba que los niños son casi inmunes al CoViD-19, noticia que pronto fue eliminada por los controles de difusión de información incorrecta de Twitter y Facebook.

En lo referente a Ecuador, en junio del 2020, la Asamblea Nacional generó un debate en torno a la eficacia del dióxido de cloro, acogiendo la posibilidad de ser un medicamento eficaz para el tratamiento de la enfermedad. No obstante, a los pocos días la Agencia Nacional de Regulación Control y Vigilancia Sanitaria informaba que no existe evidencia científica, que avale su efectividad. Por el contrario, advertía de los riesgos inminentes para la salud. Estos antecedentes demuestran una alarmante falta de información por parte de las autoridades en las que la sociedad confía (Pérez-Dasilva, Meso-Ayerdi \& Mendiguren-Galdospín, 2020), derivando en afectaciones a los medios de comunicación social y a la ciudadanía en general. Además, no ofrecen veracidad de la información y tildan las noticias con un sesgo sensacionalista e inadecuado (Tuccori et al., 2020). En consecuencia, los gobiernos centrales y locales han sido criticados por la información inexacta en torno a cómo tratar los síntomas de las enfermedades infecciosas, generando confusión social (Park, Park \& Chong, 2020). Otro problema, que genera desinformación, es la clara confrontación ideológica en los discursos políticos, afectando aún más a la problemática sanitaria, tal como ocurrió en España, donde el coronavirus sirvió como arma para la batalla entre partidos políticos (Salaverría et al., 2020). Por tanto, y bajo este escenario de enfrentamientos ideológicos e información inexacta, se requiere generar confianza desde las autoridades hacia el colectivo social para que puedan comprender las medidas sanitarias y así, disminuir la desobediencia y la propagación de casos (Moreno, Fuentes-Lara \& Navarro, 2020).

Sin duda, el rol de los medios de comunicación es fundamental para combatir la infodemia en las plataformas donde se generan espacios para el debate y discusión sobre la información falsa (Ahmed et al., 2020). El caso de éxito, en el control de la pandemia, por parte de Vietman es muy relevante para poder entender como los medios de comunicación contribuyeron a promover la conciencia social en torno a las medidas de salud pública para enfrentar el coronavirus. Asimismo, la labor del periodismo científico comunicó de manera eficaz y rápida la información de las comunidades científicas hacia la sociedad en general (La et al., 2020).

Tras los precedentes expuestos, se puede comprender que la mayoría de los estudios sobre el impacto de la infodemia han sido elaborados por investigadores del continente europeo y asiático, y muy pocos, desde la realidad de los países de América Latina, una de las regiones más golpeadas por la pandemia. Según los datos del Center for Systems Science and Engineering de la Universidad John Hopkins de Estados Unidos, con corte al 24 de julio del 2020, los países con mayor número de fallecidos por CoViD-19 a nivel latinoamericano son Perú, Brasil, Chile y Ecuador (CSSE, 2020). De esta manera, la presente investigación se centra en Ecuador por ser uno de los países a nivel mundial con más muertes producidas por esta enfermedad, ocupando el puesto $14^{\circ}$ a nivel mundial y el $4^{\circ}$ en Sudamérica. Por ello, el objetivo es analizar las prácticas comunicativas de los usuarios de los medios de comunicación en Ecuador y su percepción en torno a la información que reciben sobre el coronavirus. Además, el estudio busca identificar cuáles son los medios de comunicación o plataformas Web a las que accede la ciudadanía para informarse sobre el virus, así como examinar las temáticas informativas emergentes, analizando la credibilidad y confianza de las fuentes periodísticas, gubernamentales y las generadas a través de la interacción social en la Web 2.0. También, será relevante examinar las noticias falsas (rumores, bulos, mitos, teorías de conspiración y otros contenidos erróneos o inexactos) en torno a la pandemia a través de las redes sociales. 


\section{Metodología}

\subsection{Técnicas e instrumentos de investigación}

La metodología para el proceso de recolección de datos está basada en el método cuantitativo de carácter descriptivo. Según Sampieri (2018, p. 5), la metodología cuantitativa «representa un conjunto de procesos organizados de manera secuencial para comprobar ciertas suposiciones».

Por otro lado, para entender la apreciación del colectivo social en torno a la información que reciben sobre el coronavirus, se ha diseñado un cuestionario (https://bit.ly/2EVjgNQ) como instrumento de investigación auto-administrada a través de la plataforma Google forms. Este ha sido validado por tres docentes investigadores de la Universidad Técnica de Cotopaxi y dos de la Universidad Técnica de Ambato. Posteriormente, se ha procedido a verificar la concordancia en la respuesta de los jueces, calculando un Alfa de Cronbach de 0.898, representando un nivel alto de fiabilidad. Por su parte, Arribas (2004, p. 23) señala que «el cuestionario es un instrumento utilizado para la recogida de información, diseñado para poder cuantificar y universalizar la información y estandarizar el procedimiento de la entrevista. Su finalidad es conseguir la comparabilidad de la información en torno». De esta manera, se aplica un instrumento con 22 ítems politómicas ordinales en función de una escala de likert para identificar los medios de comunicación tradicionales (radio, prensa, televisión), digitales y plataformas Web 2.0 (redes sociales, servicios de mensajería) por las que la sociedad accede para informarse sobre el estado de la cuestión. Además, se examina el grado de credibilidad y confianza sobre la información recibida de las múltiples fuentes informativas (gubernamentales, medios de comunicación, redes sociales). Además, se pretenden determinar si la audiencia social considera haber consumido noticias falsas sobre la pandemia en redes sociales a través del análisis de las siguientes dimensiones:

a) Tipologías de medios de comunicación o plataformas Web 2.0 a las que accede la ciudadanía para informarse sobre la pandemia.

b) Temáticas informativas frecuentemente consultadas sobre el coronavirus.

c) Percepción de credibilidad y confianza en la información recibida de diversas fuentes (gubernamentales, medios de comunicación, redes sociales).

d) Percepción de noticias falsas (rumores, bulos, mitos, teorías de conspiración y otros contenidos erróneos o inexactos) en torno a la pandemia en las redes sociales.

Por último, se aplicará la ingeniería inversa de datos para recopilar reportes en Internet sobre el interés de las personas. En este caso, las temáticas informativas sobre el CoViD-19 mediante la plataforma Google Trends (Strzelecki \& Rizun, 2020). Esta herramienta en línea permite identificar la popularidad y volumen de las consultas de búsqueda mediante las palabras clave, que los usuarios introducen en el motor de búsqueda de Google (Rovetta \& Bhagavathula, 2020).

\subsection{Muestra de estudio}

En relación con el cuestionario, este se centra en un muestreo de bola de nieve exponencial, ya que permite aplicar los ítems a grupos difícilmente accesibles a causa de su posición económica o su ubicación espacial/geográfica (Alloatti, 2014). De esta manera, alcanza a estudiantes universitarios de diversa situación socioeconómica y a través de ellos, a sus familiares, comprendidos en edades desde los 18 a 65 años de todas las provincias del Ecuador. La tabla 1 ilustra el lugar de residencia de los encuestados. 
Tabla 1. Provincia de origen de los encuestados

\begin{tabular}{|l|l|l|}
\hline Provincia & Cantidad & Porcentaje \\
\hline Tungurahua & 679 & $32,5 \%$ \\
\hline Pichincha & 591 & $28,3 \%$ \\
\hline Cotopaxi & 587 & $28,1 \%$ \\
\hline Santo Domingo & 44 & $2,1 \%$ \\
\hline Guayas & 33 & $1,6 \%$ \\
\hline Napo & 25 & $1,2 \%$ \\
\hline Chimborazo & 23 & $1,1 \%$ \\
\hline Bolívar & 16 & $0,8 \%$ \\
\hline Sucumbíos & 15 & $0,7 \%$ \\
\hline Los Ríos & 10 & $0,5 \%$ \\
\hline Loja & 8 & $0,4 \%$ \\
\hline Pastaza & 8 & $0,4 \%$ \\
\hline Azuay & 7 & $0,3 \%$ \\
\hline Imbabura & 7 & $0,3 \%$ \\
\hline El Oro & 6 & $0,3 \%$ \\
\hline Galápagos & 6 & $0,3 \%$ \\
\hline Zamora Chinchipe & 6 & $0,3 \%$ \\
\hline Manabí & 5 & $0,2 \%$ \\
\hline Cañar & 4 & $0,2 \%$ \\
\hline Orellana & 3 & $0,1 \%$ \\
\hline Santa Elena & 3 & $0,1 \%$ \\
\hline Carchi & 2 & $0,1 \%$ \\
\hline Esmeraldas & 1 & $0,0 \%$ \\
\hline Morona Santiago & 1 & $0,0 \%$ \\
\hline TOTAL & 2.090 & $100 \%$ \\
\hline & & \\
\hline
\end{tabular}

Fuente: Elaboración propia

En otro orden, las temáticas informativas frecuentemente consultadas sobre el CoViD-19 son analizadas a través de los términos más populares registrados por el motor de búsqueda de Google y analizados en la plataforma de Google Trends desde el 12 de mayo al 7 de agosto del 2020. Las unidades hermenéuticas examinadas giran en torno a los conceptos de coronavirus, pandemia, CoViD-19, Covid Ecuador, coronavirus sintomas, vacuna coronavirus y Covid vacuna.

\section{Resultados}

\subsection{Acceso a la información}

Tras el análisis de la muestra seleccionada se determinó que las redes sociales son la plataforma informativa predilecta de la sociedad (20\%). En ella, se alojan los portales de los medios de comunicación tradicionales y digitales. A partir de este estudio, se pudo identificar que las personas revisan múltiples portales de noticias (15\%) con base en las plataformas Web 2.0. Asimismo, indagan en las publicaciones que son compartidas por sus familiares o amigos. En cuanto a los formatos de los medios tradicionales es evidente que lo audiovisual es impres- 
cindible para la población consultada, de ahí que la televisión (14\%) sea el tercer medio de preferencia, seguidamente de las páginas $W e b$ de los organismos de salud (12\%) en el formato clásico de la Web 1.0.

La radio $(9 \%)$ y la prensa escrita $(7 \%)$ en sus formatos tradicionales, ciertamente, ya no marcan la pauta de preferencia como fuente informativa. Finalmente, los grupos de mensajería como WhatsA$p p$, se han convertido en plataformas por donde circula la información. No obstante, no es el medio preferido para obtener noticias sobre la pandemia (6\%) (Tabla 2).

Tabla 2. Tipologías de medios de comunicación o plataformas web 2.0 a las que accede la ciudadanía para informarse sobre la pandemia

\begin{tabular}{|l|c|c|}
\multicolumn{1}{|c|}{ Tipología de plataforma o medio de comunicación } & Cantidad & Porcentaje \\
\hline Redes sociales (Facebook, Twitter, Instagram) & 1.025 & $20 \%$ \\
\hline Portales de noticias en internet de medios de comunicación tradicionales & 784 & $15 \%$ \\
\hline Televisión & 739 & $14 \%$ \\
\hline Páginas web de organismos de salud & 596 & $12 \%$ \\
\hline Portales de noticias nativos de internet & 537 & $10 \%$ \\
\hline Radio & 486 & $9 \%$ \\
\hline Páginas web oficial de los organismos del estado ecuatoriano & 373 & $7 \%$ \\
\hline Prensa escrita & 331 & $7 \%$ \\
\hline Grupos de WhatsApp & 303 & $6 \%$ \\
\hline TOTAL & $\mathbf{5 . 1 7 4}$ & $100 \%$ \\
\hline
\end{tabular}

Fuente: Elaboración propia

\subsection{Temáticas informativas emergentes sobre el coronavirus}

El 41\% de la muestra consultada afirma que la información sobre la pandemia es lo que más les preocupa frente a todas las problemáticas sociales del país (situación económica o casos de corrupción). Estos resultados se complementan con la afirmación del $70 \%$ de los encuestados que han identificado casos positivos de CoViD-19 en familiares y amigos. Por ende, la búsqueda de directrices sanitarias o información sobre el coronavirus en plataformas digitales es recurrente e importante para la ciudadanía.

En consecuencia, la investigación utiliza la herramienta Google Trends para establecer los temas informativos más consultados asociados al CoViD-19. Mediante los términos de exploración populares en torno al coronavirus en Ecuador se ha realizado una inspección en los registros a través del buscador de Google entre el 12 de mayo y 7 de agosto del 2020. La finalidad es determinar la importancia de las consultas en el transcurrir de meses y días. Para ello, se utiliza el valor cuantificable de Google Trends. que ubica en un rango de 100 al término de búsqueda más popular y de 50 a 0 al concepto medianamente popular.

Las palabras coronavirus Ecuador y Covid Ecuador son las más utilizadas para indagar información en torno a la pandemia. Sin embargo, a partir del 4 de junio comienza a posicionarse preferentemente el término de búsqueda Covid Ecuador. De estas palabras claves se desprenden consultas relacionadas a qué es el coronavirus, cómo se propaga y cuáles son sus síntomas. En el gráfico 1 se puede observar la comparativa entre la preferencia de los términos de búsqueda. 
Gráfico 1. Temática: coronavirus Ecuador y Covid Ecuador

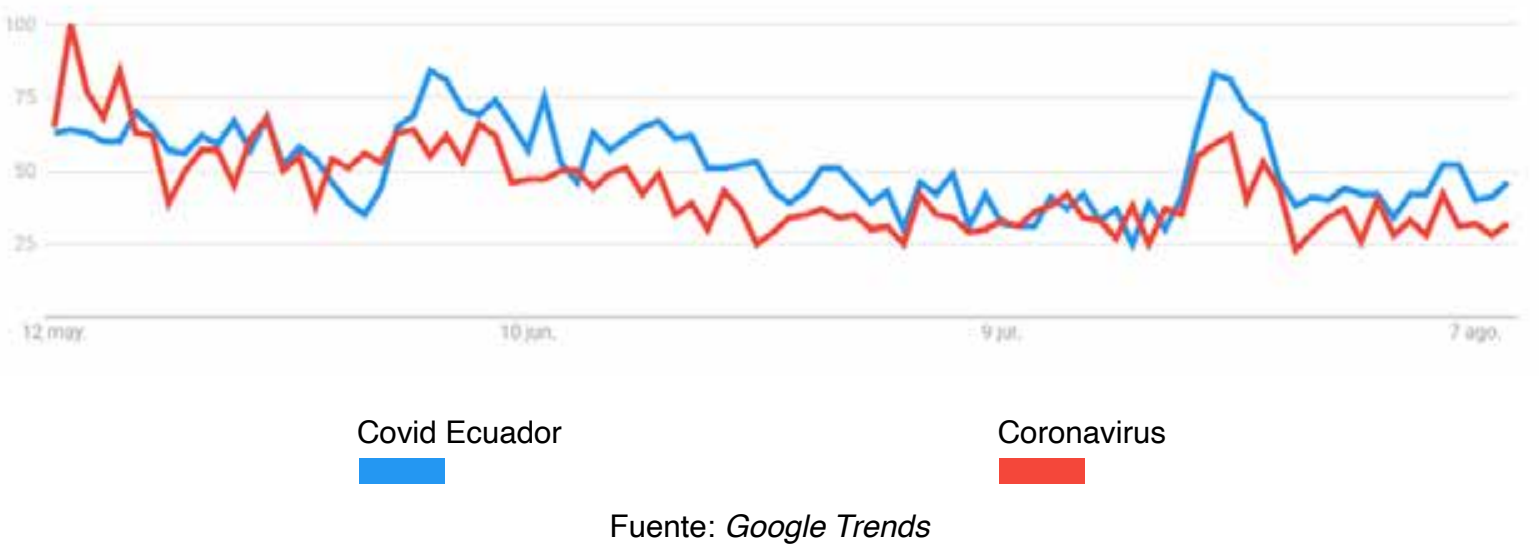

En relación con el término de búsqueda coronavirus síntomas, durante el 13 de mayo al 11 de julio del 2020 en promedio, se visualiza un rango de importancia superior a 50, mientras que a partir del 12 de julio al 9 de agosto, se evidencia una mediana importancia. Por otro lado, tratamientos con el uso de sustancias como la hidroxicloroquina y el dióxido de cloro, discutidos por líderes políticos en los escenarios mediáticos, presentan búsquedas medianamente importantes. Sin embargo, mientras la Asamblea Nacional de Ecuador discutía su uso como tratamiento para el CoViD-19, se registra una popularidad máxima, determinando el grado de influencia de los medios de comunicación y los representes políticos para posicionar una temática en la opinión pública. Respecto a esta búsqueda se identifican consultas como para qué sirven dichas sustancias y las reacciones adversas a sus componentes. Finalmente, la exploración mediante las palabras vacuna coronavirus o Covid vacuna presentan diversos rangos de importancia. Del 12 al 25 de mayo del 2020 exhibe unos resultados relevantes, seguidamente pierde importancia hasta el 14 de julio. A partir del 15 de julio vuelve a ser transcendental, asociándose a temáticas o términos de búsqueda concomitante con la Universidad de Oxford y Ruso por el anuncio del 11 de agosto en la que el presidente de Rusia, Wladimir Putin informaba sobre el registro de una vacuna contra la virus (Gráfico 2).

Gráfico 2. Temática: Vacuna coronavirus o Covid vacuna

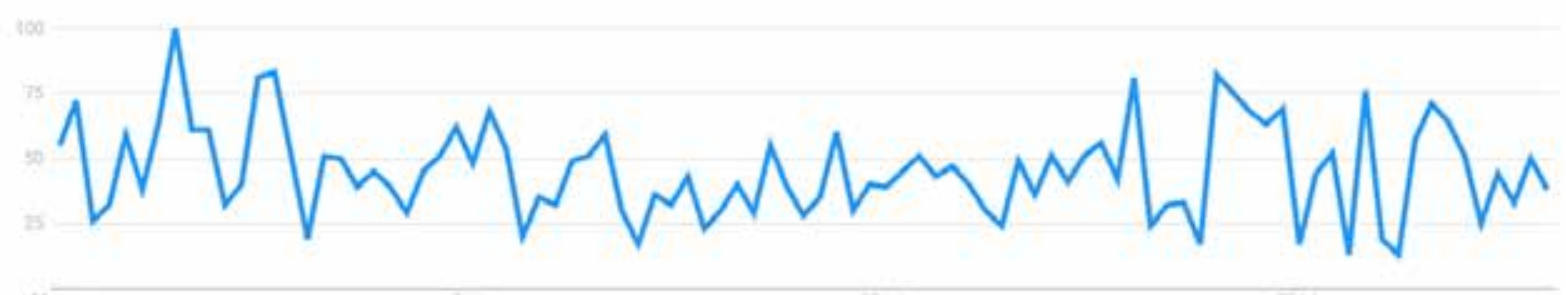

Fuente: Google Trends

Para complementar los resultados anteriores es imprescindible identificar las temáticas frecuentemente consultadas en redes sociales a través de la encuesta digital. Google Trends únicamente analiza las indagaciones realizadas a través del motor de búsqueda de Google,. De este modo, los resultados presentan similares características como el hecho de que la audiencia social está pendiente de la información de la cura o vacuna para el virus $(28,62 \%)$, así como de los síntomas, propagación de la pandemia $(27,07 \%)$ y la exploración de medicamentos para 
contrarrestar los efectos del CoViD-19 (20,40\%). La indagación de proveedores de equipos de bioseguridad $(13,68 \%)$ y alimentos $(10,23 \%)$ finalizan los temas más consultados por la muestra (Tabla 3).

Tabla 3. Temáticas informativas consultadas en redes sociales

\begin{tabular}{|l|r|c|}
\hline Opción & $\begin{array}{c}\text { Número de } \\
\text { respuestas }\end{array}$ & Porcentaje \\
\hline Información sobre la cura o vacuna para el coronavirus & 985 & $28,62 \%$ \\
\hline Información sobre los síntomas y la propagación de casos de coronavirus & 932 & $27,07 \%$ \\
\hline Información sobre un medicamento para contrarrestar los efectos del coronavirus & 702 & $20,40 \%$ \\
\hline Proveedores de equipos de bioseguridad como mascarillas, gel antibacterial & 471 & $13,68 \%$ \\
\hline Proveedores de alimentos & 352 & $10,23 \%$ \\
\hline TOTAL & 3.442 & $100 \%$ \\
\hline
\end{tabular}

Fuente: Elaboración propia

\subsection{Credibilidad y calidad de las fuentes informativas}

Para identificar la credibilidad y confianza en la información recibida, el presente estudio realiza una clasificación de fuentes informativas: a) medios de comunicación, b) canales gubernamentales y c) redes sociales de amistades y familiares.

\section{a) Medios de comunicación}

Con referencia a la credibilidad existen criterios divididos: por un lado, las personas que consideran que la información de los medios de comunicación nunca $(36,37 \%)$ o pocas veces es verdadera $(19,28 \%)$ y por otro lado, aquellas que estiman que las notas periodísticas son siempre $(25,93 \%)$ o casi siempre verdaderas $(8,13 \%)$, a pesar de la desconfianza por su veracidad $(10,29 \%)$. Por tanto, es evidente que existe una pérdida de credibilidad en una gran parte de la muestra (Tabla 4).

Tabla 4. Credibilidad de los medios de comunicación

\begin{tabular}{|l|c|c|}
\hline \multirow{2}{*}{ Percepción } & $\begin{array}{c}\text { Número de } \\
\text { respuestas }\end{array}$ & Porcentaje \\
\hline Nunca verdadera & 760 & $36,37 \%$ \\
\hline Siempre verdadera & 542 & $25,93 \%$ \\
\hline Pocas veces verdadera & 403 & $19,28 \%$ \\
\hline Algunas veces no verdadera & 215 & $10,29 \%$ \\
\hline Casi siempre verdadera & 170 & $8,13 \%$ \\
\hline Total & $\mathbf{2 . 0 9 0}$ & $100,00 \%$ \\
\hline
\end{tabular}

Fuente: Elaboración propia

En cuanto a la confianza en las tipologías de medios de comunicación, se puede destacar la preferencia por la televisión internacional $(28,89 \%)$, nacional $(21,15 \%)$ y los portales de noticias en Internet (25,74\%). Seguidamente, las redes sociales (13,21\%) cobran relevancia. A pesar de ser las principales plataformas por las cuales la ciudadanía recibe información, no gozan de veracidad. En cuanto a la radio $(5,41 \%)$ y la prensa escrita $(5,60 \%)$ se confirma la perdida de preferencia y por ende, la confianza de la audiencia (Tabla 5). 
Tabla 5. Confianza en los medios de comunicación

\begin{tabular}{|l|c|c|}
\hline Tipología de medios & Número de respuestas & Porcentaje \\
\hline Televisión internacional & 604 & $28,89 \%$ \\
\hline Portales de noticias en internet & 538 & $25,74 \%$ \\
\hline Televisión nacional & 442 & $21,15 \%$ \\
\hline Redes sociales & 276 & $13,21 \%$ \\
\hline Prensa escrita & 117 & $5,60 \%$ \\
\hline Radio & 113 & $5,41 \%$ \\
\hline Total & $\mathbf{2 . 0 9 0}$ & $100,00 \%$ \\
\hline
\end{tabular}

Fuente: Elaboración propia

\section{b) Canales gubernamentales}

La percepción de credibilidad del manejo comunicacional por parte del Gobierno ecuatoriano es mayoritariamente deficiente. Más de la mitad de las personas encuestadas consideran que los portavoces de la información no tienen experticia para manejar la pandemia $(50,10 \%)$. Por otro lado, se considera que las estrategias comunicacionales han sido buenas para enfrentar la pandemia $(29,30 \%)$. De la misma forma, existen criterios que hacen referencia a una mala dirección comunicacional $(16,50 \%)$ y escasa aprobación en la escala de muy bueno (4,10\%) (Tabla 6).

Tabla 6. Credibilidad del manejo comunicacional por parte del Gobierno ecuatoriano

\begin{tabular}{|l|c|c|}
\hline Percepción & Número de respuestas & Porcentaje \\
\hline Deficiente & 1050 & $50,10 \%$ \\
\hline Bueno & 610 & $29,30 \%$ \\
\hline Malo & 344 & $16,50 \%$ \\
\hline Muy bueno & 86 & $4,10 \%$ \\
\hline Total & $\mathbf{2 . 0 9 0}$ & $100,00 \%$ \\
\hline
\end{tabular}

Fuente: Elaboración propia

En torno a la confianza en la información que publican los organismos estatales, la muestra considera que no han revelado toda la verdad principalmente en las cifras de contagiados y fallecidos por coronavirus $(38,90 \%)$. Referente a las recomendaciones para enfrentar la pandemia a criterio de los encuestados han sido confusas sin claridad (32,60\%). Además, consideran que las directrices han sido claras y oportunas para afrontar la emergencia $(11,20 \%)$. Sin embargo, reflexionan que la información remitida agravó la emergencia sanitaria creando alarma y desorden social $(11,70 \%)$ (Tabla 7$)$.

Tabla 7. Confianza en la información que publica el Gobierno

\begin{tabular}{|l|c|c|}
\hline Ítem & $\begin{array}{c}\text { Número de } \\
\text { respuestas }\end{array}$ & Porcentaje \\
\hline No ha revelado toda la verdad & 812 & $38,90 \%$ \\
\hline Ha sido confusa sin claridad en las directrices a seguir & 682 & $32,60 \%$ \\
\hline Ha sido oportuna en las directrices para afrontar adecuadamente la emergencia sanitaria & 234 & $11,20 \%$ \\
\hline Ha creado una alarma y desorden social & 244 & $11,70 \%$ \\
\hline Clara y adecuada & 118 & $5,60 \%$ \\
\hline Total & $\mathbf{2 . 0 9 0}$ & $100,00 \%$ \\
\hline
\end{tabular}

Fuente: Elaboración propia 


\section{c) Redes sociales de amistades y familiares}

La información que comparten amistades y familiares en redes sociales genera mayor confianza $(48,28 \%)$, aunque existe un número importante de la muestra que considera que es falsa $(19,86 \%)$. Sin embargo, de manera general se destaca la credibilidad que tiene la interacción familiar y de amistad en el ecosistema digital para poder aceptar una información como verídica, superando en credibilidad a la información remitida por los medios de comunicación y las organizaciones gubernamentales $(15,50 \%)$ (Tabla 8$)$.

Tabla 8. Confianza y credibilidad en la información que publican amistades y familiares en redes sociales

\begin{tabular}{|l|c|c|}
\hline Percepción & Número de respuestas & Porcentaje \\
\hline Verdadera & 1.009 & $48,28 \%$ \\
\hline Falsa & 415 & $19,86 \%$ \\
\hline Casi siempre verdadera. & 324 & $15,50 \%$ \\
\hline Ocasionalmente verdadera & 189 & $9,04 \%$ \\
\hline Casi nunca verdadera & 153 & $7,32 \%$ \\
\hline Total & $\mathbf{2 . 0 9 0}$ & $100,00 \%$ \\
\hline
\end{tabular}

Fuente: Elaboración propia

Por último, la mayoría de la muestra consultada considera haber identificado noticias falsas, contenidos engañosos o inexactos de manera frecuente $(43,40 \%)$ o muy frecuente $(30,50 \%)$ en redes sociales, siendo compartidas en algunos casos por familiares o amigos. A pesar de ello, la confianza y la credibilidad en la información recibida por las amistades en la Red es superior a las fuentes periodísticas y gubernamentales. De manera que las fake news pueden mayoritariamente estar siendo generadas por la interacción social en la Web 2.0. (Tabla 9).

Tabla 9. Percepción de contenido engañoso

\begin{tabular}{|l|c|c|}
\hline Ítem & Número de respuestas & Porcentaje de repuestas \\
\hline Frecuentemente & 907 & $43,40 \%$ \\
\hline Muy frecuentemente & 637 & $30,50 \%$ \\
\hline Ocasionalmente & 469 & $22,40 \%$ \\
\hline Nunca & 53 & $2,60 \%$ \\
\hline Raramente & 24 & $1,10 \%$ \\
\hline Total & $\mathbf{2 . 0 9 0}$ & $100,00 \%$ \\
\hline
\end{tabular}

Fuente: Elaboración propia

\section{Discusión y conclusiones}

En una sociedad ecuatoriana hiperconectada, las redes sociales y los portales en Internet de los medios de comunicación nativos y tradicionales se han convertido en las plataformas preferidas para obtener información en torno a la pandemia, situación similar a la que ocurre en España, donde el consumo de noticias se ha centrado en los medios digitales como protagonistas (Lázaro-Rodríguez \& Herrera-Viedma, 2020) En cuanto a los medios tradicionales es perceptible la pérdida de audiencia en sus formatos clásicos, en particular la televisión mantiene niveles de preferencia baja para el consumo informativo. Sobre este medio en concreto, (Casero-Ripolles, 2020) considera que en un contexto informativo complejo y arriesgado el público opta por fuentes de larga trayectoria, así la televisión en España presenta mayores niveles de consumo 
y credibilidad. En el caso de Ecuador la televisión internacional tiene mayor veracidad que la nacional, que es superada por los portales de noticias en Internet. Por consiguiente, a nivel general, los medios de comunicación ecuatorianos presentan una pérdida de confiabilidad en una gran parte de la población, que piensa que el manejo editorial tiene intereses económicos o políticos. De ahí, la importancia de promover en la esfera pública el compromiso de los medios con la libertad de expresión y el respeto que deben tener con la audiencia que requiere información veraz por encima de sus modelos de negocio (Cetina-Presuel \& Martínez-Sierra, 2019). En cuanto a las temáticas que más preocupan a los ciudadanos se encuentran la urgente necesidad de una cura o vacuna para el coronavirus, los síntomas, la propagación de casos y los medicamentos para contrarrestar los efectos de la pandemia, estas intranquilidades surgen a raíz del aumento de casos positivos de CoViD-19 en familiares y amigos del $70 \%$ de encuestados. De esta manera las personas indagan en la Web 2.0 y en los medios de comunicación para informarse principalmente sobre los síntomas y el tratamiento para el coronavirus. De ahí que es imprescindible establecer planes de comunicación permanentes en medios de comunicación gubernamentales, privados, públicos, comunitarios y digitales que brinden la información que demanda la ciudadanía.

La gestión de la comunicación gubernamental muestra índices negativos en cuanto a la confiabilidad, donde la mayoría de los ciudadanos consideran que no se ha revelado toda la verdad y que la información ha sido confusa. No obstante, la información que publican amistades y familiares en redes sociales goza de mayor confianza a pesar de que la mayoría de la muestra consultada cree haber identificado en las redes sociales contenidos engañosos o inexactos. Frente a esto, es fundamental que la academia desde las carreras de comunicación realice su aporte a través de la infodemiología y la construcción de proyectos, que permitan la alfabetización mediática, fomentando en la sociedad un pensamiento crítico y una responsabilidad digital al momento de compartir información en redes sociales.

Tras lo expuesto, hay que destacar que es importante llevar a cabo un trabajo articulado entre Gobierno, medios comunicación, academia y la participación ciudadana (Hua \& Shaw, 2020) para mejorar el impacto de la información confiable y contrarrestar la infodemia, reforzando el conocimiento sobre la pandemia (síntomas, medicamentos, propagación de casos, vacuna) y el discurso informativo con mensajes de salud pública en multicanales tradicionales y digitales. Estos canales estarán destinados a aumentar las estrategias de prevención (Chesser, Drassen-Ham $\&$ Keene-Woods, 2020) con recomendaciones adaptadas a la realidad de cada grupo social, principalmente adecuando la información a las necesidades de los colectivos vulnerables (Liu et al., 2020). Con respecto a la pérdida de confianza en los organismos estatales, es indispensable que las autoridades no sean únicamente los portavoces de las estrategias, sino más bien, deben ofrecer la oportunidad a los profesionales de los organismos de salud y científicos de las instituciones de Educación Superior con el objetivo de generar un discurso fuerte y proactivo sobre la pandemia (Abd-Alrazaq et al., 2020) en las páginas de organismos de sanidad estatal de la Web 1.0 (páginas Web) y la $W e b 2.0$ (redes sociales) para que sean las principales opciones en el registro de información de calidad en los motores de búsqueda (Hernández-García \& Giménez-Júlvez, 2020). La revolución tecnológica ha convertido a la audiencia social en creadores y receptores de contenido (prosumer), en este sentido es clara la incidencia de este tipo de usuarios para transmitir información muchas de las veces no verificada y que contribuye a viralizar la infodemia. Por tanto, es urgente generar propuestas interactivas dirigidas a un ciudadano prosumer en la esfera digital y en los medios de comunicación para la colaboración en la identificación de información falsa mediante la alfabetización mediática. Finalmente para futuras investigaciones se plantea indagar en las razones por las que la ciudadanía no confía en la información de los medios de comunicación y en los organismos estatales. 


\section{Bibliografía}

Abd-Alrazaq, A., Alhuwail, D., Househ, M., Hamdi, M., \& Shah, Z. (2020). Top Concerns of Tweeters During the COVID-19 Pandemic: Infoveillance Study. Journal of Medical Internet Research, 22(4), 1-9. https://doi.org/10.2196/19016

Ahmad, A. R., Murad, H. R., \& Gardner, M. R. (2020). The Impact of Social Media on Hyped Panic during the COVID-19 Pandemic: The Iraqi Kurdistan Case. JMIR Mental Health, 22, 1-11. https://doi.org/10.2196/19556

Ahmed, W., Vidal-Alaball, J., Downing, J., \& Seguí, F. L. (2020). COVID-19 and the 5G conspiracy theory: Social network analysis of twitter data. Journal of Medical Internet Research, 22(5), 1-9. https://doi.org/10.2196/19458

Andreu-Sánchez, C., \& Martín-Pascual, M.-Á. (2020). Fake images of the SARS-CoV-2 coronavirus in the communication of information at the beginning of the first CoViD-19 pandemic. El Profesional de La Información, 29(3), 1-11. https://doi.org/10.3145/epi.2020. may.09

Aguaded, I., \& Romero-Rodríguez, L. M. (2015). Mediamorfosis y desinformación en la infoesfera: Alfabetización mediática, digital e informacional ante los cambios de hábitos de consumo informativo. Education in the Knowledge Society, 16(1),44-57. https://bit. ly/3b7qDPe

BBC News (2020, 3 de agosto). Coronavirus: el mapa que muestra el número de infectados y muertos en el mundo por covid-19. https://bbc.in/32jwk8n

Bonnet, J. L., \& Rosenbaum, J. E. (2020). "Fake news," misinformation, and political bias: Teaching news literacy in the 21 st century. Communication Teacher, 34(2), 103-108. https://doi.or $\mathrm{g} / 10.1080 / 17404622.2019 .1625938$

Brainard, J., \& Hunter, P. R. (2020). Misinformation making a disease outbreak worse: outcomes compared for influenza, monkeypox, and norovirus. Simulation, 96(4), 365-374. https://doi. org/10.1177/0037549719885021

Casero-Ripolles, A. (2020). Impact of CoViD-19 on the media system. Communicative and democratic consequences of news consumption during the outbreak. El Profesional de La Información, 29(2), 1-11. https://doi.org/10.3145/epi.2020.mar.23

Catalina-García, B., Sousa, J., \& Silva-Sousa, L.-C. (2019). Consumo de noticias y percepción de fake news entre estudiantes de Comunicación de Brasil, España y Portugal. Revista De Comunicación, 18(2), 93-115. https://doi.org/10.26441/RC18.2-2019-A5

Center for Systems Science and Engineering CSSE. (2020). COVID-19 Dashboard. Johns Hopkins University (JHU). https://bit.ly/3mcEebH

Cetina-Presuel, R., \& Martínez-Sierra, J. (2019). Algoritmos y noticias: Redes sociales como editores y distribuidores de noticias. Revista De Comunicación, 18(2), 261-285. https://doi. org/10.26441/RC18.2-2019-A13

Chen, E., Lerman, K., \& Ferrara, E. (2020). Tracking Social Media Discourse About the COVID-19 Pandemic: Development of a Public Coronavirus Twitter Data Set. JMIR Public Health and Surveillance, 6(2), 1-9. https://doi.org/10.2196/19273 
Chesser, A., Drassen-Ham, A., \& Keene-Woods, N. (2020). Assessment of COVID-19

Knowledge Among University Students: Implications for Future Risk Communication Strategies. Health Education \& Behavior: The Official Publication of the Society for Public Health Education, 47(4) 540-543. https://doi.org/10.1177/1090198120931420

Effenberger, M., Kronbichler, A., Shin, J. il, Mayer, G., Tilg, H., \& Perco, P. (2020). Association of the COVID-19 pandemic with Internet Search Volumes: A Google TrendsTM Analysis. International Journal of Infectious Diseases, 95, 192-197. https://doi.org/10.1016/j. ijid.2020.04.033

Eysenbach, G. (2020). How to Fight an Infodemic: The Four Pillars of Infodemic Management. Journal of Medical Internet Research, 22(6), 1-6. https://doi.org/10.2196/21820

Hernández-García, I., \& Giménez-Júlvez, T. (2020). Assessment of Health Information About COVID-19 Prevention on the Internet: Infodemiological Study. JMIR Public Health and Surveillance, 6(2), 1-11. https://doi.org/10.2196/18717

Hua, J., \& Shaw, R. (2020). Corona virus (CoViD-19) "infodemic" and emerging issues through a data lens: The case of China. International Journal of Environmental Research and Public Health, 17(7), 1-12. https://doi.org/10.3390/IJERPH17072309

Kouzy, R., Abi Jaoude, J., Kraitem, A., el Alam, M. B., Karam, B., Adib, E., Zarka, J., Traboulsi, C., Akl, E., \& Baddour, K. (2020). Coronavirus Goes Viral: Quantifying the COVID-19 Misinformation Epidemic on Twitter. Cureus, 12(3), 1-9. https://doi.org/10.7759/cureus.7255

Lázaro-Rodríguez, P., \& Herrera-Viedma, E. (2020). Noticias sobre CoViD-19 y 2019-nCoV en medios de comunicación de España : el papel de los medios digitales en tiempos de confinamiento News on CoViD-19 and 2019-nCoV in Spanish media : The role of digital media in times of confinement. IweTel, 1-11. https://doi.org/10.3145/epi.2020.may.02

Liu, Q., Zheng, Z., Zheng, J., Chen, Q., Liu, G., Chen, S., Chu, B., Zhu, H., Akinwunmi, Ba., Huang, J., Zhang, C. J. P., \& Ming, W. K. (2020). Health communication through news media during the early stage of the CoViD-19 outbreak in China: Digital topic modeling approach. Journal of Medical Internet Research, 22(4), 1-12. https://doi.org/10.2196/19118

Lotero-Echeverri, G., Romero-Rodríguez, L. M., \& Pérez-Rodríguez, M. A. (2018). Fact-checking vs. Fake news: Periodismo de confirmación como recurso de la competencia mediática contra la desinformación. index.comunicación, 8(2), 295-316.

Masip, P., Aran-Ramspott, S., Ruiz-Caballero, C., Suau, J., Almenar, E., \& Puertas-Graell, D. (2020). Consumo informativo y cobertura mediática durante el confinamiento por el CoViD-19: sobreinformación, sesgo ideológico y sensacionalismo. El Profesional de La Información, 29(3), 1-12. https://doi.org/10.3145/epi.2020.may.12

Mejia, C. R., Ticona, D., Rodríguez-Alarcon, J. F., Campos-Urbina, A. M., Catay-Medina, J. B., Porta-Quinto, T., Garayar-Peceros, H., Ignacio-Quinte, C., Esteban, R. F. C., Ruiz Mamani, P. G., \& Tovani-Palone, M. R. (2020). The media and their informative role in the face of the coronavirus disease 2019 (COVID-19): Validation of fear perception and magnitude of the issue (MED-COVID-19 ). Electronic Journal of General Medicine, 17(6), 1-6. https://doi.org/10.29333/ ejgm/7946

Moreno, A., Fuentes-Lara, C., \& Navarro, C. (2020). CoViD-19 communication management in Spain: Exploring the effect of information-seeking behavior and message reception in public's evaluation. El Profesional de La Información, 29(4), 1-16. https://doi.org/10.3145/epi.2020.jul.02 
Naeem, S. B., \& Bhatti, R. (2020). The Covid-19 'infodemic': a new front for information professionals. Health Information \& Libraries Journal, 24(1), 62-68. https://doi.org/10.1111/ hir. 12311

Odriozola-Chéné, J., Díaz-Noci, J., Serrano-Tellería, A., Pérez-Arozamena, R., Pérez-Altable, L., Linares-Lanzman, J., García-Carretero, L., Calvo-Rubio, L. M., Torres-Mendoza, M., \& AntónBravo, A. (2020). Inequality in times of pandemics: How online media are starting to treat the economic consequences of the coronavirus crisis. El Profesional de La Información, 29(4), 1-16. https://doi.org/10.3145/epi.2020.jul.03

Organización Mundial de la Salud (Ed.) (2020). Actualización de a la Covid-19. https://bit. ly/332vHzh

Park, H. W., Park, S., \& Chong, M. (2020). Conversations and Medical News Frames on Twitter: Infodemiological Study on COVID-19 in South Korea. Journal of Medical Internet Research, 22(5), 1-11. https://doi.org/10.2196/18897

Pérez-Dasilva, J.-A., Meso-Ayerdi, K., \& Mendiguren-Galdospín, T. (2020). Fake news y coronavirus: detección de los principales actores y tendencias a través del análisis de las conversaciones en Twitter. El Profesional de La Información, 29(3), 1-22. https://doi.org/10.3145/ epi.2020.may.08

Pulido, C. M., Ruiz-Eugenio, L., Redondo-Sama, G., \& Villarejo-Carballido, B. (2020). A New Application of Social Impact in Social Media for Overcoming Fake News in Health. International Journal of Environmental Research and Public Health, 17(7), 24-30. https://doi.org/10.3390/ ijerph17072430

Pulido, C. M., Villarejo-Carballido, B., Redondo-Sama, G., \& Gómez, A. (2020). COVID-19 infodemic: More retweets for science-based information on coronavirus than for false information. International Sociology, 35(4), 377-392. https://doi. org/10.1177/0268580920914755

Rinaldi, L. (2020). La task force per le fake news sul CoViD-19 tra protezione del diritto all'informazione e ipotesi di censura del web. BioLaw Journal, 8, 653-665. https://bit.ly/3jMfJQt

Rovetta, A., \& Bhagavathula, A. S. (2020). COVID-19 -related web search behaviors and infodemic attitudes in Italy: Infodemiological study. Journal of Medical Internet Research, 22(5), 1-10. https://doi.org/10.2196/19374

Romero-Rodríguez, L., de-Casas-Moreno, P., \& Torres-Toukoumidis, A. (2016). Dimensions and indicators of the information quality in digital media. [Dimensiones e indicadores de la calidad informativa en los medios digitales]. Comunicar, 49, 91-100. https://doi.org/10.3916/C49-2016-09

Romero-Rodríguez, L., de-Casas-Moreno, P., Caldeiro-Pedreira, M. (2018). Desinformación e infoxicación en las cuartas pantallas. En Ignacio Aguaded (Ed.), Competencias mediáticas en medios digitales emergentes (pp. 73-92). Comunicación Social ediciones y publicaciones.

Salaverría, R., Buslón, N., López-Pan, F., León, B., López-Goñi, I., \& Erviti, M.-C. (2020). Desinformación en tiempos de pandemia: tipología de los bulos sobre la CoViD-19 . El Profesional de La Información, 29(3), 2020-2022. https://doi.org/10.3145/epi.2020.may.15

Sampieri, R. H. (2018). Metodología de la investigación: las rutas cuantitativa, cualitativa y mixta. McGraw Hill.

Sterrett, D., Malato, D., Benz, J., Kantor, L., Tompson, T., Rosenstiel, T., Sonderman, J., \& Loker, 
K. (2019). Who Shared It?: Deciding What News to Trust on Social Media. Digital Journalism, 7(6), 783-801. https://doi.org/10.1080/21670811.2019.1623702

Strzelecki, A., \& Rizun, M. (2020). Infodemiological Study Using Google Trends on Coronavirus Epidemic in Wuhan, China. International Journal of Online and Biomedical Engineering (IJOE), 16(04), 139-146. https://doi.org/10.3991/ijoe.v16i04.13531

Tangcharoensathien, V., Calleja, N., Nguyen, T., Purnat, T., D’Agostino, M., Garcia Saiso, S., Landry, M., Rashidian, A., Hamilton, C., AbdAllah, A., Ghiga, I., Hill, A., Hougendobler, D., van Andel, J., Nunn, M., Brooks, I., Sacco, P. L., de Domenico, M., Mai, P., ... Briand, S. (2020). A Framework for Managing the COVID-19 Infodemic: Methods and Results of an Online, Crowdsourced WHO Technical Consultation. Journal of Medical Internet Research, 22, 1-8. https://doi.org/10.2196/19659

Toffler, A. (1980). La tercera ola. William Morrow

Torres-Toukoumidis, Á.; Romero-Rodríguez, L.M.; de-Casas-Moreno, P. y Aguaded, I. (2017). Construcción del discurso bélico desde las agencias internacionales de noticias: Estudio de Caso Atentados del 13 de noviembre de 2015. Revista Mediterránea de Comunicación/Mediterranean Journal of Communication, 8(1), 121- 135. https://www.doi.org/10.14198/MEDCOM2017.8.1.9

Tuccori, M.; Convertino, I.; Ferraro, S.; Cappello, E.; Valdiserra, G.; Focosi, D.; Corrado, B. (n.d.). The Impact of the COVID-19 "Infodemic" on Drug-Utilization Behaviors: Implications for Pharmacovigilance. Drug Safety, 4, 699-709. https://doi.org/10.1007/s40264-020-00965-w 